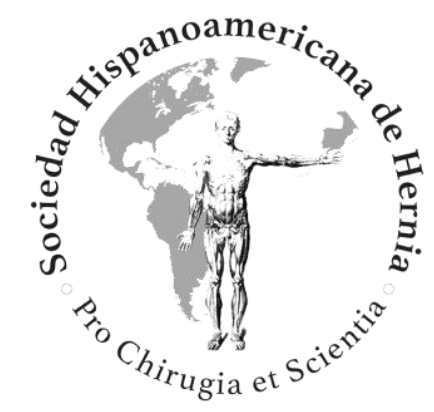

Hernia inguinoescrotal masiva de contenido vesical

\title{
Massive inguino-scrotal bladder hernia
}

10.20960/rhh.00382

$10 / 28 / 2021$ 


\section{OR 382}

\section{Hernia inguinoescrotal masiva de contenido vesical}

Massive inguino-scrotal bladder hernia

Emilio Prieto Díaz Chávez , Raúl Ochoa Gómez², José L. Medina Chávez³, Sergio G. Prieto Díaz Chávez² , Juan Carlos Mayagoitia González ${ }^{4}$

Departamentos de ${ }^{1}$ Cirugía, ${ }^{2}$ Urología y ${ }^{3}$ Quirófano. Clínica San Francisco. Colima (México). ${ }^{4}$ Hospital Médica Campestre. León, Guajanato (México)

Recibido: 20-10-2020

Aceptado: 05-11-2020

Autor para correspondencia: Emilio Prieto Díaz Chávez. Departamento de Cirugía. Clínica San Francisco. C/ Venustiano Carranza, 369. 28000 La Atrevida. Colima (México)

Correo electrónico: epdch59@gmail.com

DOI: $10.20960 /$ rhh.00382

Conflictos de interés: los autores declaran no tener conflictos de interés.

\section{RESUMEN}

Introducción: Aunque la hernia inguinal es una patología con una alta incidencia entre la población general, es raro encontrar la participación de la vejiga dentro del saco herniario, que solo tiene una incidencia de entre el 0.5 y el $3 \%$. El objetivo de este estudio es informar de un caso de hernia inguinoescrotal con contenido de vejiga y revisar la literatura relevante. 
Caso clínico: Paciente masculino de 69 años con una masa inguinoescrotal izquierda, con micción espontánea de la porción intraabdominal de la vejiga y cuyo vaciado completo requiere de presión suprapúbica manual de la hernia y elevación escrotal para completarla (signo de Mery). La cirugía evidenció un defecto del suelo inguinal directo de tipo IV de Gilbert a través del que protruye un segmento importante de la vejiga que cruza el suelo del canal inguinal. Se repara de forma convencional con la técnica de Lichtenstein con malla de polipropileno pesada.

Conclusión: Entidad poco frecuente que, por lo general, constituye un hallazgo transoperatorio cuando no tenemos estudios previos de imagen y que se resuelve con una hernioplastia convencional con uso de material protésico.

Palabras clave: Hernia inguinoescrotal, contenido vesical, hernia vesical.

\section{ABSTRACT}

Introduction: Although inguinal hernia is common in the general population, the bladder involvement within the hernial sac is rare, and its incidence varies between 0.5 and $3 \%$. The aim of this study is to report a case of inguinoscrotal hernia with bladder content and to review the relevant literature.

Case report: A 69-year-old male patient was clinically assessed for a left inguinoscrotal mass and spontaneous urination of the intraabdominal portion of the bladder with the need for manual suprapubic pressure of the hernia and scrotal elevation to complete the bladder emptying (Mery's sign). Surgery revealed a direct Gilbert type IV inguinal floor defect, with a significant bladder segment protrusion across the floor of the inguinal canal. The defect was successfully 
repaired in a conventional way with a Lichtenstein technique employing heavy polypropylene mesh.

Conclusion: A rare entity that generally constitutes an intraoperative finding when we do not have previous imaging studies, and that is resolved with a conventional hernioplasty using prosthetic material.

Keywords: Inguinoscrotal hernia, bladder content, bladder hernia.

\section{INTRODUCCIÓN}

El deslizamiento de la vejiga dentro del saco de una hernia inguinal se define como hernia vesical. Las primeras publicaciones sobre la hernia inguinoescrotal con contenido vesical propiamente dichas se remontan a Guy de Chauliac, en $1363^{1-3}$. Sin embargo, el primer caso debidamente reportado fue por parte de Felix Platter en $1550^{3,4}$. Aunque para 1986 Thompson y cols. ${ }^{5}$ presentaron un enfoque de tratamiento unificado para 75 casos, los informes posteriores generalmente se limitan a casos aislados, incluso cuando se presenta una hernia inguinoescrotal con contenido vesical masivo, lo que demuestra la rareza de esta afección, o por lo menos, la dificultad de diagnóstico en el preoperatorio.

Las hernias vesicales se clasifican según su relación con el peritoneo en paraperitoneales, si el peritoneo recubre su cara externa (60\%), que pueden ser directas e indirectas; extraperitoneales, con una frecuencia inferior, caracterizadas como defectos de pequeño tamaño y no recubiertos por el peritoneo (32\%), e intraperitoneales, las menos frecuentes (4\%), pues toda la vejiga herniada está recubierta por el peritoneo. Son siempre indirectas y suelen reducirse con facilidad ${ }^{6,7}$. La hernia inguinoescrotal con contenido vesical es mucho menor ${ }^{10,11}$ : la hernia vesical inguinal de tipo paraperitoneal o cistocele escrotal es una patología poco frecuente, con una incidencia de entre el 0.5 y el $3 \%$, que alcanza hasta el $10 \%$ en pacientes mayores de 50 años ${ }^{7-9}$. No 
obstante, la incidencia de hernias inguinoescrotales con contenido vesical masivo (herniación de más del 50 \% de la vejiga) es mucho menor $^{10,11}$. Los principales factores etiopatogénicos son las deformidades vesicales, particularmente las adquiridas, así como la debilidad de la pared abdominal en el canal inguinal. También se han asociado otros factores, como la obesidad y la hipertensión abdominal ${ }^{6}$. Presentamos el caso clínico de un paciente con este tipo de hernia poco frecuente. Realizamos una revisión de la bibliografía relacionada.

\section{CASO CLÍNICO}

Paciente masculino de 69 años que se presentó a la consulta externa de cirugía con antecedentes de hipertensión arterial sistémica en control. Antecedente de trombosis venosa profunda izquierda ocho meses antes en el miembro pélvico izquierdo, manejada con acenocumarina y posteriormente con clopidrogrel, que no dejó secuelas de ningún tipo. Posteriormente, presentó dolor intenso en la rodilla izquierda, que necesitó infiltración intraarticular, sin especificar etiología. Además, refirió tener antecedentes de sintomatología urinaria con cuadros de retención urinaria intermitente por patología prostática.

Durante el interrogatorio refirió cuadro actual con molestias en la región inguinal izquierda con irradiación al miembro pélvico contralateral (derecho), así como un aumento del volumen continuo y progresivo en la región inguinal mencionada. Micción parcial espontánea. El vaciado vesical total solo se completaba con la presión manual suprapúbica de la hernia y la elevación del escroto (signo de Mery).

En la exploración física: peso de $95 \mathrm{~kg}$ y talla de $1.69 \mathrm{~cm}$. La palpación abdominal no reveló visceromegalias, tumoraciones ni puntos dolorosos. Sobre la región inguinal izquierda se identificó un gran saco herniario parcialmente reductible, doloroso a las maniobras de movilización. La región inguinal derecha, sin presencia de saco herniario. En el examen 
rectal, la próstata se encontró con un aumento de tamaño, compatible con hipertrofia adenomatosa benigna de grado II/IV.

Se solicitó una tomografía axial computarizada abdominal que evidenció un defecto herniario en la región inguinal izquierda, con un cuello de 5.8 $\mathrm{cm}$ por donde se producía un prolapso de la vejiga urinaria en un $80 \%$, de $15 \mathrm{~cm}$ de largo, que ocupa todo el hemiescroto izquierdo (figs. 1,2).

Se programó de forma electiva la hernioplastia inguinal por abordaje abierto bajo cloqueo peridural. En la exploración quirúrgica de la región inguinal izquierda se identificó un defecto del piso inguinal (hernia directa de tipo IV de Gilbert/Rutkow), por el que protruía un segmento grande de la vejiga que pasaba a través del conducto inguinal, acompañaba al cordón espermático, se prolongaba hacia el escroto y alcanzaba los recesos inferiores de la bolsa escrotal (fig. 2).

La vejiga deslizada se disecó del escroto y de los elementos del cordón en su totalidad y volvió a colocarse a través del defecto en su posición anatómica normal a través de una disección circuncidante de base de la hernia y de la invaginación del contenido herniario mediante una sutura continua de Vicryl ${ }^{\circledR}$, del músculo transverso y oblicuo menor al ligamento inguinal para la contención de la vejiga y del saco herniario en el espacio preperitoneal ante la ausencia de bordes de fascia transversalis para este efecto. Posteriormente, se completó una hernioplastia inguinal con una técnica convencional de Lichtenstein, con una malla plana de polipropileno pesado, fijada lateralmente con una sutura de Prolene ${ }^{\circledR} 2 / 0$ al ligamento inguinal y medialmente al tendón conjunto con puntos separados con Vicryl ${ }^{\circledR} 2 / 0$ (fig. 3 ). No se presentaron incidentes transoperatorios.

La convalecencia transcurrió sin incidentes y se resolvieron los síntomas urinarios de micción incompleta. El paciente fue dado de alta 36 horas después. Solo se reportó como complicación temprana, en el día 30 del posquirúrgico, una retención aguda de orina secundaria a su prostatismo y la presencia de una infección de las vías urinarias, 
resuelta sin cateterización de la vejiga, solo con antibioticoterapia selectiva contra el microorganismo encontrado en el urocultivo.

El seguimiento del paciente se prolongó tres años completos, sin que se reportarán complicaciones tardías ni recidivas, ya que falleció por una causa ajena a su patología herniaria (infección por COVID-19).

\section{DISCUSIÓN}

Aunque la hernia inguinal es una patología con una alta incidencia entre la población general, es poco frecuente encontrar la participación de la vejiga dentro del saco herniario: tiene una incidencia de entre el $0.5 \%$ y el $3 \%$ de todas las hernias, y llega hasta el $10 \%$ en hombres mayores de 50 años ${ }^{7-9}$.

Los factores predisponentes para la formación de estas hernias son: una pared abdominal deficiente, una morfología anormal de la vejiga, ya sea congénita o adquirida, y la presencia de distensión constante de la vejiga causada por el síndrome de obstrucción infravesical de cualquier origen. La causa más frecuente es la obstrucción prostática benigna crónica. Otros factores que contribuyen a su desarrollo son la edad, los lipomas prevesicales y las hernias inguinoescrotales grandes ${ }^{1,2,5,7,8,13}$.

Un dato importante en las hernias inguinoescrotales grandes o gigantes es que favorecen la compresión de la vena femoral ocasionada por el tamaño y el contenido del saco herniario, lo que condiciona la aparición de trombosis de esta vena como antecedente, tal como lo describe $\mathrm{Baca}^{14}$ en su reporte de una hernia inguinoescrotal gigante, de la misma forma en que ocurrió como antecedente en nuestro paciente.

La mayoría de los pacientes con hernias inguinoescrotales masivas con contenido vesical son asintomáticos, y estas hernias se descubren incidentalmente durante la reparación de la hernia inguinal $\left.\right|^{3,8,12,13}$. Cuando se presenta una hernia vesical masiva, como en el caso que describimos, el síntoma predominante es la micción en dos etapas (signo de Mery) ${ }^{3,6,11}$. González ${ }^{8}$, en su reporte de cuatro casos con 
diagnóstico preoperatorio, comenta que se basa en el síntoma primordial de vaciamiento incompleto con ayuda manual posterior, y muchos serán hallazgos transoperatorios. Sin embargo, también reporta que habrá un porcentaje de pacientes con hernias de segmentos vesicales que no serán identificados tampoco durante el transoperatorio, pero encuentra que la mayoría de estos tendrán algunos síntomas posoperatorios, como disuria, tenesmo o retención urinaria, que pueden indicarnos que el paciente presenta deslizamiento no identificado de la vejiga durante la hernioplastia ${ }^{8}$.

Los síntomas más frecuentes presentados por nuestros pacientes concuerdan con los reportados en la literatura, como polaquiuria, micción en dos etapas (signo de Mery), disuria, masa escrotal, hematuria aislada y, con menor frecuencia, nicturia, tenesmo vesical y retención aguda de orina ${ }^{3,6,11}$.

En cuanto a la exploración física abdominal, se presenta con mayor frecuencia una protrusión de contenido abdominal a través del orificio inguinal superficial que, en la mayoría de las veces, es parcialmente reductible o francamente irreductible. No hay datos físicos en la exploración que sugieran la presencia de una hernia con contenido vesical.

El diagnóstico por imagen ante la sospecha de la presencia de un segmento de vejiga en el saco herniario puede realizarse a través de una TAC, una urografía excretora e, idealmente, con una cistografía retrógrada ${ }^{8}$. En el caso que presentamos, la tomografía computarizada con contraste oral e intravenoso aportó datos sobre el estado de ambos sistemas excretores, así como sobre las relaciones de la vejiga en su trayecto herniario tal como lo define Conde $^{7}$ en su reporte de caso clínico (fig. 3).

La hernioplastia habitual con técnica sin tensión es el único tratamiento de elección para este tipo de pacientes. El abordaje preferido debe ser el abierto, que ofrece la ventaja de poder reducir con mayor facilidad el 
segmento de vejiga herniada. La reparación laparoscópica también ha sido reportada por Khan y cols. ${ }^{4}$, quienes informaron de una serie de dos pacientes sometidos a una reparación laparoscópica de hernias de vejiga. La laparoscopia ofrece las ventajas generales de los accesos mínimos en comparación con los abordajes abiertos, pero representa una dificultad importante en el momento de la reducción de un gran saco herniario junto con la vejiga, en especial cuando existen múltiples adherencias al escroto y al cordón espermático. Si se requiriese una resección parcial de la vejiga, el abordaje abierto representa una menor dificultad técnica ${ }^{4}$.

Las dos opciones quirúrgicas para el manejo de la vejiga en el transoperatorio son el reposicionamiento quirúrgico de la vejiga herniada (reducción) o la resección de la vejiga herniada en caso de estrangulación y gangrena o presencia de un segmento sumamente extenso en el que se sospeche dificultad para su reducción o malfuncionamiento después de restituir un segmento con distonía permanente; en uno u otro caso, siempre junto a la reparación protésica del suelo inguinal $2^{2,3,5,13,15}$.

En varones adultos con herniaciones de vejiga, el trígono permanece fijo y es la única porción vesical que conserva su localización habitual ${ }^{2}$, por lo que se prefiere la reducción de la vejiga a la resección de la porción herniada, en la medida en que la primera no solo no requiere la incisión de la pared vesical, obviando la necesidad de suturar la vejiga, además de realizar una cistostomía, con lo que se evita así la contaminación del campo quirúrgico ${ }^{1,2,5,12}$. La resección de la vejiga se realiza en casos que cursan con necrosis de la vejiga, una hernia de cuello de menos de $0.5 \mathrm{~cm}$ de diámetro o un divertículo o tumor de vejiga $^{12}$.

La técnica quirúrgica empleada en nuestro paciente para la reparación de esta hernia inguinoescrotal masiva de tipo deslizante con contenido vesical fue similar a la de una reparación estándar para la hernia 
inguinal, en la mayoría de las cuales se utiliza una reparación sin tensión tipo Lichtenstein, optando por reducir la vejiga herniada con reposición quirúrgica ${ }^{4,13,14}$ (figs. 4,5).

\section{CONCLUSIÓN}

La hernia inguinoescrotal con contenido vesical es una entidad poco frecuente, generalmente encontrada como hallazgo transoperatorio cuando no se realizaron estudios previos de imagen. En particular, debe considerarse en hombres obesos, mayores de 50 años, que presentan un aumento del volumen intermitente del área inguinal o escrotal acompañado de síntomas del tracto urinario inferior, principalmente vaciado incompleto de vejiga y necesidad de maniobras externas para completar su vaciamiento total. El manejo quirúrgico es el convencional en cualquier hernia inguinal.

\section{BIBLIOGRAFÍA}

1. Bechara AJ, Rovegno Agustín R, D'Osvaldo C, Mocellini Iturralde JA, Fernández $\mathrm{H}$. Hernias vesicales inguinoescrotales presentacion de un caso y revision del tema. Rev Argentina Urología. 1988;54(2):45-9.

2. Juan Escudero JU, Ramos de Campos M, Ordoño Domínguez F, Fabuel del Toro M, Serrano de la Cruz Torrijos F, Navalón Verdejo P, et al. Hernias vesicales inguinoescrotales. Arch Esp Urol. 2007;60(3);31-6. DOI: 10.4321/S0004-06142007000300002

3. Ríos-Hernández N. Signo de Mery en paciente con hernia vesicalinguinoescrotal. Presentación de caso. Anales de Radiología México. 2018;17(1):73-7. DOI: 10.24875/ARM.M18000009

4. Khan A, Beckley I, Dobbins B, Rogawski KM. Laparoscopic repair of massive inguinal hernia containing the urinary bladder. Urol Ann. 2014;6:159-62. DOI: 10.4103/0974-7796.130654

5. Thompson JE Jr, Taylor JB, Nazarian N, Bennion RS. Massive inguinal scrotal bladder hernias: a review of the literature with 2 new 
cases. J Urol. 1986;136(6):1299-301. DOI: 10.1016/s00225347(17)45321-3

6. Ignacio-Morales CV, Aragón-Tovar AR, Torres-Medina E, Itzcoatl Muñoz-Islasd E, Vilchis-Cárdenas MA. Hernia vesical. Informe de un caso y revisión de la bibliografía. Rev Mex Urol. 2010;70(5):293-5.

7. Conde Sánchez JM, Espinosa Olmedo J, Salazar Murillo R, Vega Toro P, Amaya Gutiérrez J, et al. Hernia Vesical Inguinoescrotal Gigante. Caso Clínico y Revisión de la Literatura. Actas Urol Esp. 2001;25(4):315-9. DOI: 10.1016/S0210-4806(01)72623-X

8. González-Urquijo M, Mayagoitia-González JC, Rodarte-Shade M, Bermea-Mendoza JH, Gil-Galindo G. Large inguinal bladder hernias: can a preoperative diagnosis be made? Hernia. 2019;23(6):1221-7. DOI: 10.1007/s10029-019-01955-3

9. Delgado-Pereira B. Hernia vesical inguinal. Boletín de la Sociedad de Cirugía del Uruguay. 1963;34(1-2).

10. Casamayor MC, Sanz Ji, Bono A, Ligorred La, Baque F, Esclarín Ma, Abad J. Fracaso renal agudo secundario a hernia vesical inguino-escrotal. Actas Urol Esp 1999;23(2):171-5.

11. Merino Peñacoba LM, Ortiz de Solórzano Aurusa J, Juárez Martín ME, González Perea JG, Beltrán de Heredia Rentería J. Hernia vesical masiva y signo de Mery. A propósito de un caso. Rev Hispanoam Hernia. 2015;3(2):71-4.

12. Hamidi Madani A, Mohammadi Nikouei H, Baghani Aval H, Enshaei A, Asadollahzade A, Esmaeili S. Scrotal herniation of bladder: a case report. Iran J Med Sci. 2013;38(1):62-4.

13. Foladi N, Farzam F, Tahir Aien M. Massive inguino-scrotal herniation of urinary bladder in an infant (scrotal cystocele)-case report. Radiol Case Rep. 2020;15(5):607-9. DOI: 10.1016/j.radcr.2020.02.024

14. Baca PJE, Domínguez CLG. Hernia gigante inguinoescrotal. Rev Gastro Mex. 2016;6:1-3. DOI:10.1016/j.rgmx.2016.06.003 
15. Wang $P$, Huang $Y$, Ye J, Gao G, Zhang F, Wu H. Large sliding inguino-scrotal hernia of the urinary bladder: $A$ case report and literature review. Medicine (Baltimore). 2018;97(13):e9998. DOI: 10.1097/MD.0000000000009998

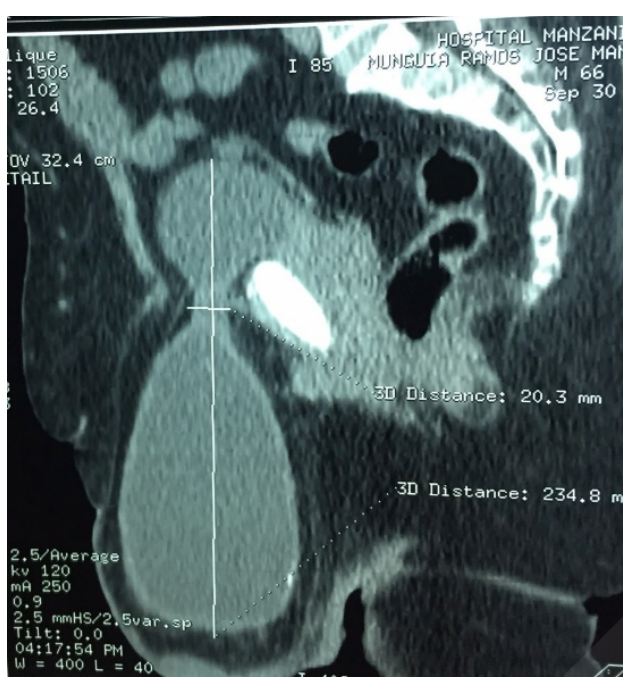

Figura 1. Tomografía axial computarizada. En el corte sagital se observa aproximadamente el $80 \%$ de la vejiga pasando a través del canal inguinoescrotal izquierdo.

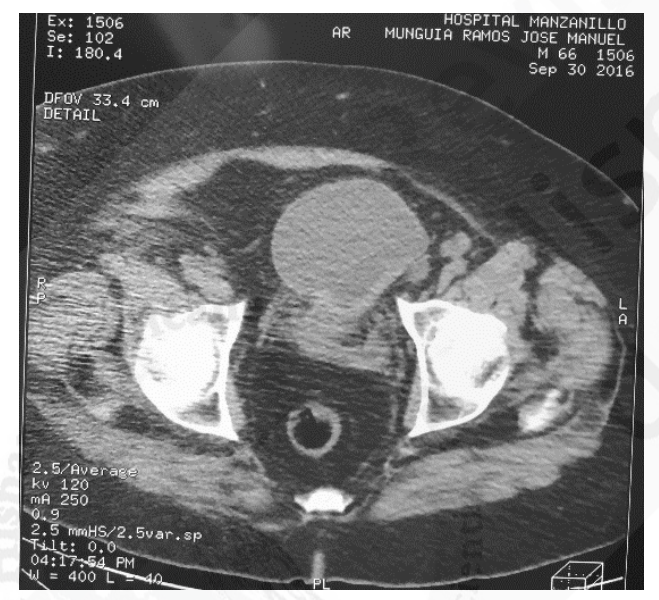

Figura 2. En corte axial muestra una imagen ovoide con interior hipodenso, que deforma el canal inguinal izquierdo. 


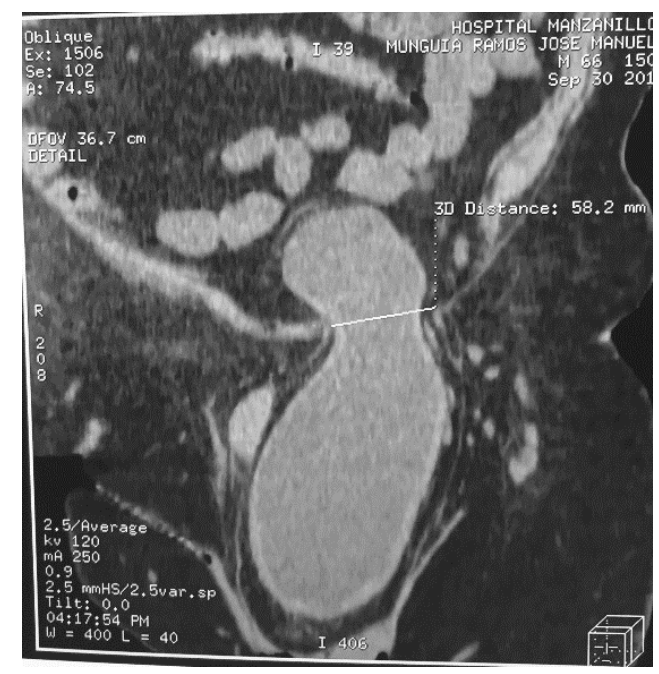

Figura 3. Tomografía axial computarizada que muestra la hernia inguinoescrotal con contenido vesical masivo con desviación de la vejiga hacia el lado de la hernia.

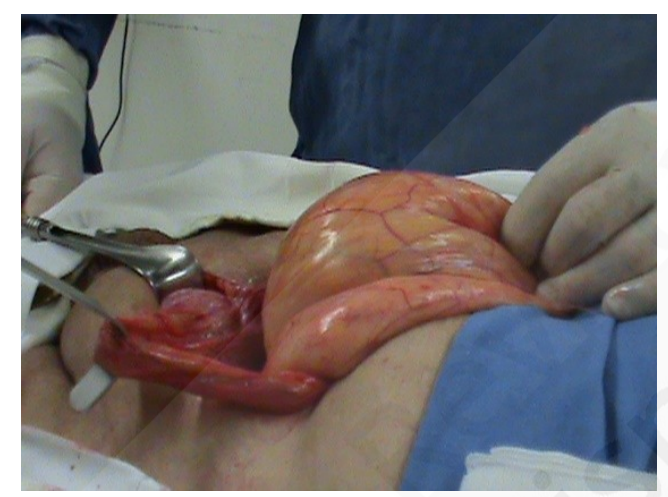

Figura 4. Hernia directa con un gran saco herniario que contiene la vejiga.

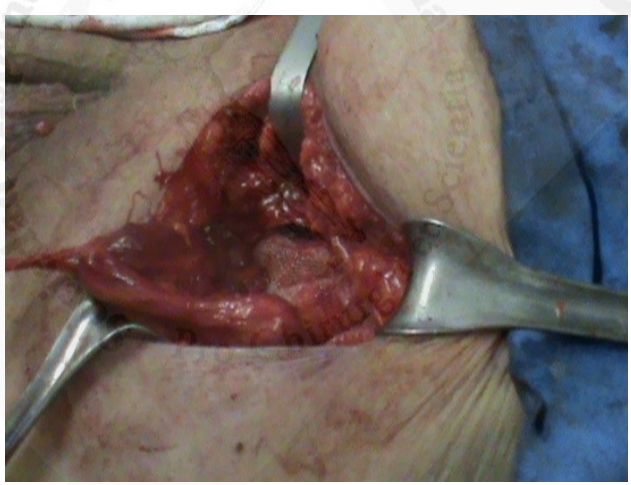


Figura 5. Reparación con malla de polipropileno utilizando la técnica de Lichtenstein. 